\title{
Understanding Data Analytics Practices in Kansas City: The Importance of Multi-Level Stewardship, Collaborative Governance and an Incremental Strategy
}

\author{
Felippe A. Cronemberger \\ University at Albany, State University of New York \\ fcronemberger@albany.edu
}

\author{
J. Ramon Gil-Garcia \\ University at Albany, State University of New York \& \\ Universidad de las Americas Puebla \\ jgil-garcia@ctg.albany.edu
}

\begin{abstract}
Local governments face complex challenges and are increasingly pressured to find innovative strategies to address them. Recently, they are leveraging data analytics and a number of policy modeling techniques to respond to those challenges. While a lot of attention is given to smart initiatives and data analytics endeavors in big cities, not enough studies have looked at experiences of smaller jurisdictions, who also have to solve difficult and often relatively unique problems. This paper examines how Kansas City, Missouri (KCMO) is currently using data analytics as a means of creating useful information for problem-solving around the city. As part of the What Work Cities network, the city embraced data-driven management as a new modus operandi and has been recognized nationally as a successful case. Among the main findings, it can be highlighted that: (1) data analytics can be kick started by committed public leadership, but is enabled organically by stewards who have traditionally and iteratively responded to information needs from a variety of local government agencies; and (2) stakeholders and organizations that are involved with data analytics have different capabilities, face different challenges, and frequently adopt incremental strategies that include data management and governance aspects.
\end{abstract}

Keywords: Data Analytics, Data-driven

Management, Leadership, Local

Governments, Organizational Capabilities,

Policy-Making

\section{Introduction}

Cities across the globe are being constantly challenged by problems that are both difficult and diverse. Traditional concerns such as emergency preparedness [1] now coexist with the need to manage and govern technologies and the data they produce as those cities attempt to become more effective and overall smarter [2]. Adequate response to those challenges often requires information sharing and collaboration at multiple levels [3], as well as the ability to apply technologies to problem-solving [1]. While research has consistently identified the need for more and better research on local governments' particularities and specific needs [4], few empirical studies have scrutinized the many elements that are part of smartness in government, including, for example, the central role data play in enabling better decisions [5]. One of the reasons is that smart city is a multidimensional topic [6], which makes it hard to consider all the relevant aspects when studying or developing a smart strategy.

In more than a decade of research about smart cities, multiple studies have asserted that technology and governance models are critical to push smart cities forward, but similar attention has not been given to the role of data use and data analytics practices in creating practical results to local government officials and the citizens they serve [7]. In particular, little is known about (1) how those practices take place in small and medium cities, (2) what affects public servants' routine in achieving their analytical goals, and (3) what challenges they routinely face [4], [8], [9].

This paper contributes to addressing this gap by analyzing the experience of a city that has been engaging in the use of data analytics in a variety of public policy fronts: Kansas City, Missouri (KCMO). Since its acceptance into Bloomberg's What Works Cities initiative in 2015, KCMO has become a national leader in the use of data and analytics for innovative problem-solving [10]. Specifically, KCMO has achieved important milestones in the use of data, from identifying urban factors influencing a decaying infrastructure to establishing new standards of excellence in problem reporting through their 311 call center services.

Taking a lessons learned approach, this study empirically explores data analytics practices in a local government context. It addresses three questions: what appears to influence data analytics use in Kansas City, Missouri? How KCMO developed new capabilities 
through data analytics practices? What practical lessons could be learned from this case that could be useful to other cities with similar experiences? This paper proposes answers to these questions by presenting research on both micro, individual-level aspects of data analytics, such as leadership and data handling, and macro, organizational level aspects, such as data governance and management. In the light of the literature presented, we explain to what extent the case fits in or deviates from what is known in the current literature.

The paper is organized in seven sections, including the foregoing introduction. Section two includes a review of recent literature, covering topics such as data governance and management. A conceptual model is also presented. Section three introduces the methods used for data collection and analysis as well as the dimensions that guided the qualitative data analysis. Section four introduces the KCMO case, setting the stage for the empirical findings that are presented in section five and discussed in section six. Section seven provides concluding remarks, some implications of this study, and future research directions.

\section{Data Governance, Management and Analytics: An Overview}

Data analytics has been generally referred to as a set of techniques and methodologies, including modeling and simulation [11], pattern recognition [12], and business intelligence practices [13]. Holding the promise of supporting insight creation and datadriven problem solving [11], not rarely those insights are expected to come from increasing amounts of data [9], [11] and will help managers in identifying opportunities to drive or improve organizational performance [14]. It has also been highlighted that collecting and cleaning data [7] as well as combining and integrating data from multiple sources, is a fundamental part of the data analytics process [7], [11], [14]. In terms of its goals, research suggests that data analytics appears to create value in different fields and domains, from urban design [15] to cybersecurity [16].

Data analytics could also encompass modeling techniques that are not only restricted to information processing technologies, but also to collaborative practices. According to Luna-Reyes and colleagues, modeling approaches [17], such as group model building [18] are known to enable problem-solving through meeting sessions and artifacts that consider both qualitative and quantitative approaches as valid sources of data. Other authors shed light to information science and management research on boundary objects [19], artifacts that facilitate collaboration and are capable of bringing members of interdisciplinary teams to common grounds [20]. As literature suggests, collaboration is a central part of data analytics practices [21] by helping supporting sustainable information creation, management, and analysis endeavors [22].

Factor-oriented research on data analytics, one that considers the specifics of its use and specific issues in local governments is relatively scarce, and remains highlighted as needed [4], [7], [11]. That need contrasts with technological hopes that, quite often, do not offer practical responses to information management and data governance problems that have been known for decades to compromise efficiency in public organizations [23]. In realms outside of public administration and management, research has also been focused on identifying data analytics or analytical capabilities [24]. The specifics of data use in government and the factors that are known to influence data analytics endeavors are explained in the following sections.

\subsection{Data Handling and Management}

Ways of handling data at the analytical level are commonly referred to as data cleaning and wrangling. These preliminary steps are known to be critical before running analysis, since they affect the quality of data [25] and the insights to be derived from them [26]. The effective use of data analytics is contingent to a number of pre-processing practices, such as data harvesting and aggregation [27]. This echoes traditional challenges in government such as data integration [28], a concern that can be specially daunting for smart city efforts. According to Lawati and Barbosa [29], data's richness and quality are essential to enable intelligence practices and improve policy decision-making. From a more subjective perspective, value and usefulness [30] are also important aspects of data use, particularly in attempts to generate insights [31].

Finally, organizations dedicated to becoming more data-driven often set out to manage data through technologies - Big data and IoT (Internet of Things), for example - and face additional challenges, many of them related to their adoption and use by different stakeholders. To Brous and colleagues [32], challenges include limited capability in managing technological assets and difficulties in incorporating data into decision-making processes, which stem, among other factors, from poor management of expectations about outcomes. The complexity of those challenges showcase the hurdles in institutionalizing data management and sustaining the organizational use of data and its necessary infrastructures [33]. Not rarely, data management issues can also be examined 
through data governance lenses, particularly in interorganizational settings. In those environments, sociotechnical complexity is the norm, and having interoperable systems [34] in place becomes both a goal and a means of achieving data management and data governance objectives. Important overlaps between management and governance in creating enabling environments for data use makes identification of the problem difficult [35]. Conceptual distinctions and the role of governance are discussed next.

\subsection{Data Governance in perspective}

At the organizational level, data handling is expressed in broader concerns such as the necessity of managing data to achieve goals or govern them so multiple instances and stakeholders can benefit from them [36]. This field has been broadly discussed as data or information governance [26], [36].In essence, data governance aims to increase "the value of data and minimize data-related costs and risks" [37]. To Brous, Janssen, and Vilminko-Heikkinen [38], "Data governance provides organizations with the ability to ensure that data and information are managed appropriately, providing the right people with the right information at the right time". In addition, the need to maximize positive effects of information sharing and integration [23] and interoperable data exchanges [39] is consistent with the paradigm that orchestration of work is key in complex inter-organizational environments [40].

Governance is interdisciplinary and its nature demands research to account for structures and mechanisms when assessing the way organizations function [41]. Its study calls for epistemic approaches that assume that problems are "mutually implicated" [42]. This socio-technical complexity requires, among many things, defining roles and responsibilities [43] and establishing clear decision-making processes and frameworks that "ensure effective management of IT [44].

Research on data governance, in specific, has explored data architectures and systems dedicated to technical availability of data, such as data lakes [36], fast data [45], data marts and warehouses [46], and big data infrastructures [47], or to how stakeholders articulate around such artifacts through proper management and collaboration [48] and engage in coproduction practices to improve service delivery to citizens [5]. In general, research is concerned with enabling and enhancing decision and policy making in fronts such as improving data science service delivery [49] increasing transparency [11], or achieving specific governance goals [25], [47]. According to Priebe and Markus [50], "harmonization" of responsibilities is central to data warehouses implementation, and consequently, data governance success. Similarly, Dremel, Stoeckli, and Wulf [51] have observed that proper coordination of sociotechnical components is key to leverage the use of data analytics solutions.

To benefit from such endeavors, such as in fostering data science practices, authors found that organizations should be able to examine more closely the data and the contexts in which it is managed, analyzed, and used. According to Passi and Jackson [52], for example, models and existing knowledge are as important to analytics as trust from management that is indeed a worthy initiative, a view that is also echoed by other researchers [36]. To Löfgren and Webster [25], perceptions on quality and the ownership of data are critical in conducting data analytics, while to van den Broek and van Veenstra [48] centralizing collaborative efforts around large amounts of data may both reduce risk and foster innovation.

In the context of innovative practices in the public sector, the use of data is contingent to an ecosystem view, in which openness, diffusion and a shared vision are key to orchestrate data use in local governments [40]. This ecosystem view is similar to the work of Amini, Imteaj, and Pardalos [53], for whom data analytics and data science practices can be leveraged through interdependent networks through which information gets exchanged and used. The authors suggest that an "interdependent data analytics framework", if successfully implemented and developed, can optimize decision-making and improve the quality of decision-making processes. Ecosystems and frameworks for data governance are also discussed in the context of stewardship, that is, mechanisms that facilitate data access and use and enable actions for informed decision-making [54].

\subsection{A Data Management-Governance Framework for Data Analytics Use}

From different perspectives, literature suggests that handling, management and governance are part of the same data process. Acting in different stages, each component is expected to be critical to drive effective data analytics use (Figure 1). At one level or another, the absence of one may compromise the overall success of data analytics use. This study will analyze $\mathrm{KCMO}$ experience in the light of these influencing factors and will discuss the main findings. 


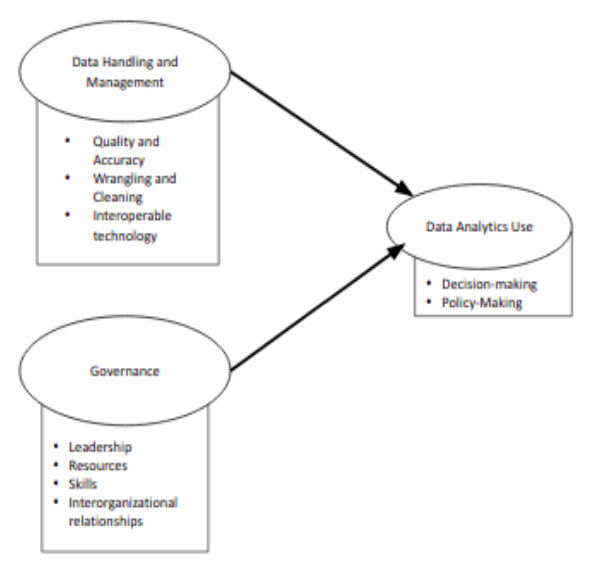

Figure 1. Data Management and Governance as influencing factors for Data Analytics Use

\section{Research Design and Methods}

This paper analyzes semi-structured interviews conducted with fifteen members from different agencies of the city government in Kansas City, Missouri. The interviews were conducted in the Spring of 2017 and most took place in offices in the City Hall or in agencies in the vicinity. All respondents were already familiar with data analytics practices being conducted in their own projects or in projects they were part of. Backgrounds were diverse and included information technology, project management, engineering, data science and policy, and human resources. The interviews lasted from 15 minutes to 50 minutes and involved open-ended questions in four areas: 1) Data and Information; 2) Technology Infrastructure; 3) Organizational and Institutional aspects; 4) External factors. This framework mirrors research in digital government [55], one that focuses on exploring socio-technical phenomena in a comprehensive manner, without losing track of details that can emerge from the richness of qualitative data. This approach is especially enlightening when the intricacies of organizational and institutional aspects need to be uncovered and related as determinants of complex governance arrangements [56].

Data and information questions were focused on the use that is made of data and information in the staff daily routine. Respondents were asked what issues they try to address with data analytics and what challenges they face in the process. For example, questions such as "How do you get access to the data you need?" and "Could you please elaborate on any data-related challenges you encounter when conducting data analytics practices?" were asked. Technology questions directed the conversation towards the analytical technologies being used and the role of supporting infrastructure in achieving data analytics goals. Organizational and institutional questions asked respondents to elaborate on the dynamics of organizational interactions and on factors that influence the ability to conduct data analytics and effectively use the outputs. Among other questions, respondents elaborated on their perception of leadership and management and the extent to which resources were available. Lastly, the respondents were asked about their thoughts on aspects external to their organization, for example, how citizens' perceived their efforts, the quality of inter-organizational relationships and the political and economic climate.

\section{The KCMO case}

In June 2016, Kansas City, Missouri, joined the What Works Cities initiative, a program started by Bloomberg philanthropies and dedicated to help local governments across the country improve their use of data. Cities that started in WWC are now part of a nationwide network, where experiences on challenges and lessons learned are exchanged and used as a benchmark for other cities. Among the goals and many outlined objectives of the program are engaging residents with data resources and improving the quality of decision-making by public leadership [10].

While not existing at the time interviews for this project were conducted, a certification program is now in place to assess successes in the realm of data use. The program verifies the extent to which WWC are able to understand their data and effectively use them to make decisions [10] . Cities that complete the assessment become eligible to receive support from WWC and its partners and remain on the initiative's radar to receive orientation to continue to improve.

KCMO emerged as a leader in data-driven practices to "make decisions, set priorities, build trust, and engage residents" [57]. As of early 2018, there were several accomplishments such as reduction in unemployment, residential development, and a street car that is fostering economic activity downtown; all of it as a result of the city leadership's commitment with the use of facts, data, and evidence to make decisions [58].

According to Bloomberg Cities [59], KCMO successfully promoted a cultural shift to data-driven management, institutionalizing the use of data to "make decisions, measure results, and hold leaders accountable". This institutionalization is made evident by several mandates: 1 ) the KCMO's data ordinance, an initiative that "requires the city manager to develop and maintain an infrastructure to data reporting" [59]; 2 ) the KCStat program, where the mayor and the city manager would meet with department leaders to check 
if goals are being met; and 3) the establishment of the DataKC, formerly the Office of Performance Management, that is now formalized as the house of City Hall's data experts [59]. It has been observed that DataKC, in particular, evolved organically from the City's 311 call center, responding to organizational needs such as "process improvement" and "survey administration" [59].

Prior to becoming DataKC, the call center perceived itself as an organization that does "data for the city" and "performance metrics". As leaders started to embrace data analytics use more systematically and the demand for their work increased, it became critical to people involved with data analytics in KCMO that they define their scope of action to themselves as well as to internal stakeholders and external audiences they serve. Such scope was mainly data related, but it is also focused on building capabilities for other departments through collaboration and training [59]. Partnering with programs would occur in areas such as "customer feedback", "data management", "continuous improvement", and "data storytelling".

\section{Main Findings}

This section presents the main results of the study. Most findings were aspects of data and information use as well as organizational and institutional factors. External factors were also mentioned in the interviews, but largely connected to data and information needs or organizational goals. The following sections briefly describe some of them.

\subsection{Collaboration for Data Collection, Management, and Analytics}

Organizations involved in data analytics use in KCMO's had multiple and distinct goals and objectives. For example, while the Office of Human Resources has performance and developmental goals for public servants, the Department of Public works is dedicated to designing and maintaining city infrastructure. Such a multitude of goals led to specific data needs and distinct ways of collecting data and managing it.

Collecting data, especially new data, required going beyond departmental boundaries and existing data sets. Two reasons for that were highlighted by the interviewees. The first one was the fragmented nature of existing datasets. Many of them were scattered across legacy systems - some of which had not been used and became outdated. The second reason is that questions that started to be prioritized by the Mayor's Office became increasingly more complex, either requiring a more extensive survey of existing datasets or the involvement of technological divisions, such as the Information Technology department. Those divisions are asked to improve or to think of systems, devices and smart ways of collecting data - either through sensors or by changing or updating existing platforms for data manipulation. At the time data for this study were collected, the OpendataKC repository was in place, but, few interviewees said they were using any of the resources from there, mainly because their current information needs did not require any of the datasets made available. When asked on whether they foresaw its use to address such a need, the vast majority said yes, with many mentioning that they would eventually look into that repository.

When not relying on existing technological infrastructure, data analytics practitioners would either conduct the best analysis possible with available data or think of incremental strategies to collect more data or improve the quality of existing datasets. Data collection processes relied on extensive collaboration among city departments and units, even across departments that were not directly associated with a given goal. In some cases, however, some of the goals of partnering organizations converged in terms of city operations - for example, the Department of Parks and Recreation and the Health Department collaborated to collect data to study neighborhood demographics. One of the opportunities for synergy was to understand to what extent neighborhood access to parks affected health indicators in the region. In this case, a common goal for data analytics use was perceived to be mutually useful, and synergy helped moving the initiative forward.

While several organizations observed that efforts to collaborate on data analytics were being put in place, levels of engagement varied across agencies. At an inter-organizational level, respondents observed limited resources, competing priorities and the need to deliver existing projects often slowed down interorganizational collaboration. When involved in goals that were multidisciplinary and prioritized, multiple agency stakeholders seemed ready to engage in capability-building and in obtaining resources that were needed, including data.

Almost all interviewees stated that collaboration around data analytics practices is key to achieve results from an organizational and an interorganizational perspective, and were willing to support or take part in such initiatives. When asked on what factors would prevent them from engaging in collaboration, answers were sparse, and often concerning operational data collection burdens, such as getting users to input data in a certain system. There was consensus on inter-organizational level enablers, and that is detailed next. 


\subsection{Data Analytics, Collaborative Governance, and Leadership from the Top and across City Agencies}

Leadership and stewardship towards data analytics practices are mainly shared by two departments: the Office of Performance Management - now DataKC and, coordinated by the City's Chief Data Officer; and the Mayors' Office / City Management, led by the Chief Innovation Officer. While both offices would define data as being central to the activities they were conducting in City Hall, their approach to data is different. The team in charge of innovation at KCMO was mostly in charge of understanding citizens' needs, both currently and for the future. Among their responsibilities was the need to understand how a smart city vision can be embraced by KCMO. That vision would come after research on particularities of the city - citizens' concerns, demographics, urban space and existing challenges, to name a few - and would seek response in information and communication technologies that could range from implementing IoT sensors to leveraging existing ones such as mobile phones for data collection("Data is at the core of everything we do"). Interviewees also revealed that the innovative initiatives around data were sponsored and enabled by the Mayor and the City Manager, who brought together the political and operational climate to make it happen. This alignment allowed KCMO leadership to champion a vision of where KCMO needs to do to respond to present needs and future challenges.

In addition, the Office of Performance Management is concerned with data analytics in practice. Leveraging both quantitative and qualitative methods, the team would be primarily concerned with the question-asking and question-answering dynamics, conducting the analysis with rigor and with an orientation to creating insights. Among the requests, city leadership asks questions such as "What do you know about this (specific problem or policy domain)?"or "Can you create a map for this?".

Constant exposure to data questions and datarelated demands by other teams in the City Hall made organizational and inter-organizational challenges in data analytics use particularly more evident to the Office of Performance Management. According to an interviewee, "there is no data analytics without getting the data first", and that could be a real challenge. According to the interviewees, cleaning data and managing it is a central part of a process that is never actually over, mainly because of data accuracy and quality issues. That process would also include accounting for biases and for contextual information, a practice that helps the audience of data analytics outputs understand the analysis ("What do these data mean?" and "Where are we going?"). To interviewees, data helps build a narrative that fosters discussions about existing problems or programs in a "truthful" and "transparent way".

At a more strategic level, DataKC is also responsible for making data open, both to the public and to City staff, an initiative that helps overcoming red tape, and also have a faster process for formal requests and sharing information with people who may need it to respond to their own questions as demands keep coming.

\section{Discussion and Implications}

This section discusses some of our main findings and proposes a revised version of a model representing the role of data management and governance on data analytics use. Some of the findings are consistent with previous research that has identified similar factors as critical to data use in general, and to data analytics, in particular.

\subsection{The Importance of Data Management and Governance for Data Analytics}

At the operational level, the role of tasks such as cleaning data and handling data quality issues and inaccuracies was highlighted by those directly involved in data manipulation and management. The challenges associated with it were considered routine and did not appear to prevent data analytics practitioners to move forward with their analytical routines - rather, DataKC embraced the responsibility to do it and lead the way so other organizations could do it as well. This commitment with the importance of providing support and educate stakeholders on data practices was highlighted as coming from analytical skills and knowledge acquired from previous experiences such as the 311 call center. In that experience, KCMO leveraged the internal capabilities of an established data-driven unit so it could develop capabilities in other departments that were less experienced with data analytics. That suggests that data analytics capabilities are built through knowledge sharing efforts triggered by agency leaders and supported by data champions across the city government.

Data governance practices also appear to be stewarded by DataKC, - a division that has been sponsored by public leadership and remained committed to use data resources to execute a smart city vision. Interestingly, data governance did not appear to be centered at architecture and formal procedures, as much as it was in participative arrangements dedicated to addressing information needs public 
leaders have. constantly in touch with public and civil servants through KCStat meetings, both learning from citizens and calibrating goals, expectations and the vision on KCMO city development. Data governance, in this case, seems to be about ensuring data analytics outputs are accessible and transparently available for policy-makers in collaborative meetings. In contrast with defining protocols and technological architectures for data delivery, data governance's major role is supplying data analytics outputs as boundary objects that guide collaboration around public problems that need to be jointly understood, revisited, and learned.

Another interesting finding has to do with the notion of value and its association to data analytics and data itself. As covered in the literature, value is a data attribute, not rarely assessed subjectively. In KCMO, while data were often referred to as an asset, value itself was not as much in datasets but in the process of contextualizing it and producing actionable information from it. It appears to be very important to create mechanisms - stewardship mechanisms - that enrich data and enhance the value of data analytics practices and assets more holistically. As suggested by evidence, enriching data sets comes from asking important questions, acknowledging limitations of data sources and proactively collecting more data. This finding does not necessarily downplay the technical relevance of data warehouses or data lakes, but appears to highlight that iterative arrangements such as data collaboratives [21], [48] and open data ecosystems [40] are potentially a more suitable framework to study data analytics in local governments.

Also, findings clearly show that capabilities in terms of data collection and data management are not equal across multiple organizations. Despite interest in using data analytics, some organizations had fewer data resources, or not enough analysts to leverage them. Some appeared to be quite ready both in terms of data and staff, but have just started to put processes in place to use data more systematically. As sponsors of incremental practices, DataKC staff seemed attentive to those varying needs, acknowledging those differences and the impact they may bring to the city government's overall performance and goals.

Lastly, when designing programs that facilitate understanding of data analytics at an interorganizational level, it appears that an incremental approach is key, given the necessity of answering questions from different departments while navigating internal resource challenges such as data availability, quality and the analytical capability to get value out of it. It also seems clear that even for those directly involved with data, openness not only of data, but also towards methodologies and practices, is an enabler of data analytics. Complementing research on collaborative data analytics [60], these findings appear to reveal that organizations using data analytics should not only worry about data or drivers of their use, but also about factors limiting their actual use.

\subsection{An Adapted Model of Data Analytics Use, Management, and Governance}

Findings put in perspective the framework presented in the literature review (Figure 1). As evidence suggests, governance-related factors are known to influence data analytics use directly and indirectly (Figure 2). Indirectly, factors such as leadership enable data stewardship, including certain handling and management practices. Those practices were found to be particularly effective when steered by committed leadership from at least three levels of the organization. More directly, governance was again found to influence data analytics use, but, more specifically, through incremental practices and strategies in data use. Those incremental and collaborative practices, as well as experiences learned through previous successful data-related initiatives, and frequent consultation with DataKC experts, made access to specific data resources possible and actionable.

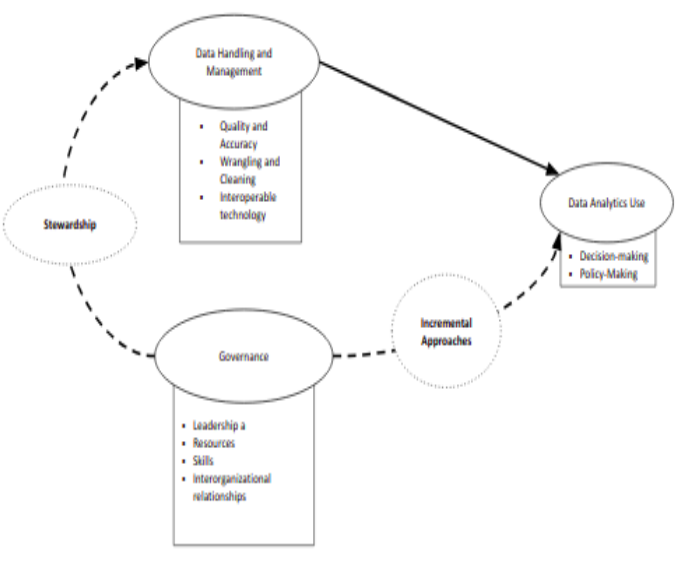

\section{Figure 2. Stewardship, Data Management, and Incremental Approaches as Governance mediators of Data Analytics Use}

Improved decision-making and policy-making, operationalized here as central goals of data analytics use, appeared to be a function of how well multi-level leadership could stimulate inter-organizational collaboration and empower intra-organizational champions to foster data analytics incremental practices. Such instrumentality would allow focusing 
on data analytics outputs and learning from the process.

It seems clear, therefore, that if local governments are to create value from data analytics use, efforts are not to be centered on data resources exclusively, but also at leveraging inter-organizational capabilities both creating the initial momentum through leaders at the top and scattered across different units in the organization, and exploring internal knowledge in incremental practices and strategies, which was the case of KCMO with prior experiences, such as 311. Acknowledging those additional elements appeared to be critical, jump-starting data analytics use both from the top and from within the city, an effort that appears to be more pervasive.

\section{Conclusion}

Evidence from the KCMO's experience contributes to refinement and expansion of current understanding about data governance and the role of leadership and stewardship in local governments. That is because KCMO's leadership did not only define the way data should be used or governed, but was also concerned with creating a vision and leveraging internal capabilities needed for a data analytics culture and vision to be built and shared across the whole city government. Leaders from across organizations clearly played a crucial role in generating momentum, but an existing staff of data and analytics savvy practitioners, already experienced with incremental practices, were instrumental in moving the initiative forward. DataKC symbolizes the work of data collectors, data managers, but, more fundamentally, of data analytics stewards who reinforced the use of evidence in decision-making and policy-making from the top and from within.

Most factors influencing data analytics practices in local governments outlined in the literature review were also found in the case. The way those factors play out in a complex inter-organizational environment such as KCMO are revealing because how an interdisciplinary and collaborative staff perceived data analytics and decided to embrace it. Embracing it, in the case, involved moving forward with practices centered around both specific data and policy problems that demanded flexibility to collaborate and adapt. Future studies should look into concepts that were found to be relevant for KCMO such as multilevel leadership, data stewardship and incremental practices and strategies, and test to what extent they are equally relevant in other government realities, outside or inside of the scope of the What Works Cities initiatives. Those concepts and dimensions did not receive enough attention in this paper, a limitation that could be addressed in future research. Future studies could also divide observations by their roles, functions and overall impact in the endeavors they were part of. They could include frameworks that are more focused on defining success of collaborative practices, and dimensions beyond the ones chosen for this paper.

There are important practical lessons to be learned in that realm and certainly a shortage of studies that explore particularities of small and medium cities, which, as outlined, are also finding smart ways of governing data resources and solving problems through data analytics. Those smart ways appear to be endogenously created, perhaps not only as a result of existing collaborative arrangements, but also of past collaborative experiences, capable of leveraging existing knowledge with data practices and applying it to ongoing policy challenges. Observing these dynamics may inform data analytics practitioners and help them to have a clearer picture of their team's readiness for analytics.

\section{References}

[1] T. Soyata, H. Habibzadeh, C. Ekenna, B. Nussbaum, and J. Lozano, "Smart city in crisis: Technology and policy concerns,” Sustainable Cities and Society, vol. 50, p. 101566, Oct. 2019.

[2] J. R. Gil-Garcia, J. Zhang, and G. Puron-Cid, "Conceptualizing smartness in government: An integrative and multi-dimensional view," Gov. Inf. Q., vol. 33, no. 3, pp. 524-534, 2016.

[3] J. R. Gil-Garcia, T. A. Pardo, and M. De Tuya, "Information Sharing as a Dimension of Smartness: Understanding Benefits and Challenges in Two Megacities,” Urban Aff. Rev. Thousand Oaks Calif, p. 1078087419843190 , May 2019.

[4] I. Mergel, R. K. Rethemeyer, and K. Isett, "Big data in public affairs,” Public Adm. Rev., vol. 76, no. 6, pp. 928-937, 2016, Accessed: Feb. 04, 2017. [Online].

[5] B. Allen, L. E. Tamindael, S. H. Bickerton, and W. Cho, "Does citizen coproduction lead to better urban services in smart cities projects? An empirical study on e-participation in a mobile big data platform," Gov. Inf. Q., vol. 37, no. 1, p. 101412, Jan. 2020.

[6] J. R. Gil-Garcia, N. Helbig, and A. Ojo, "Being smart: Emerging technologies and innovation in the public sector,” Gov. Inf. Q., vol. 31, pp. I1-I8, Jun. 2014.

[7] F. Cronemberger and J. Gil-Garcia, Problem Conceptualization as a Foundation of Data Analytics in Local Governments: Lessons from the City of Syracuse, New York. 2020.

[8] A. T. Chatfield and C. G. Reddick, "Customer agility and responsiveness through big data analytics for public value creation: A case study of Houston 311 on-demand services," Gov. Inf. Q., vol. 35, no. 2, pp. 336-347, Apr. 2018.

[9] F. Cronemberger and J. R. Gil-Garcia, "Big Data and Analytics as Strategies to Generate Public Value in Smart Cities: Proposing an Integrative Framework,” in 
Setting Foundations for the Creation of Public Value in Smart Cities, M. P. Rodriguez Bolivar, Ed. Cham: Springer International Publishing, 2019, pp. 247-267.

[10] "Home | What Works Cities - What Works Cities," What Works Cities. https://whatworkscities.bloomberg.org/ (accessed Mar. 06, 2020).

[11] L. Hagen, T. E. Keller, X. Yerden, and L. F. LunaReyes, "Open data visualizations and analytics as tools for policy-making,” Gov. Inf. Q., vol. 36, no. 4, p. 101387, Oct. 2019.

[12] R. Pérez-Chacón, J. M. Luna-Romera, A. Troncoso, F. Martínez-Álvarez, and J. C. Riquelme, "Big Data Analytics for Discovering Electricity Consumption Patterns in Smart Cities,” Energies, vol. 11, no. 3, p. 683, Mar. 2018, Accessed: Oct. 27, 2019. [Online].

[13] H. Chen, R. H. L. Chiang, and V. C. Storey, "Business Intelligence and Analytics: From Big Data to Big Impact,” Miss. Q., vol. 36, no. 4, pp. 1165-1188, 2012, Accessed: Apr. 18, 2016. [Online].

[14] C.-S. Wang, S.-L. Lin, T.-H. Chou, and B.-Y. Li, “An integrated data analytics process to optimize data governance of non-profit organization," Comput. Human Behav., vol. 101, pp. 495-505, Dec. 2019.

[15] P. P. J. Yang and Y. Yamagata, "Chapter 1 - Urban systems design: shaping smart cities by integrating urban design and systems science," in Urban Systems Design, Y. Yamagata and P. P. J. Yang, Eds. Elsevier, 2020, pp. 1-22.

[16] M. Swarnkar and R. S. Bhadoria, "Security Issues and Challenges in Big Data Analytics in Distributed Environment," in Distributed Computing in Big Data Analytics, Springer, Cham, 2017, pp. 83-94.

[17] L. F. Luna-Reyes et al., "Modeling and Simulation as Boundary Objects to Facilitate Interdisciplinary Research: Modelling and Simulation for Interdisciplinary Research,” Syst. Res. Behav. Sci., Nov. 2018, doi: 10.1002/sres.2564.

[18] P. S. Hovmand, D. F. Andersen, E. Rouwette, G. P. Richardson, K. Rux, and A. Calhoun, "Group ModelBuilding 'Scripts' as a Collaborative Planning Tool," Syst. Res. Behav. Sci., vol. 29, no. 2, pp. 179-193, 2012/03//Mar/Apr2012, Accessed: Sep. 01, 2015. [Online].

[19] A. P. Spee and P. Jarzabkowski, "Strategy tools as boundary objects,” Strategic Organization, vol. 7, no. 2, pp. 223-232, 2009, Accessed: Dec. 19, 2016. [Online].

[20] C. Oswick and M. Robertson, "Boundary Objects Reconsidered: from Bridges and Anchors to Barricades and Mazes,” Journal of Change Management, vol. 9, no. 2, pp. 179-193, Jun. 2009, Accessed: Dec. 19, 2016. [Online].

[21] I. Susha, M. Janssen, and S. Verhulst, "Data collaboratives as 'bazaars'? A review of coordination problems and mechanisms to match demand for data with supply,” Transforming Government: People, Process and Policy, vol. 11, no. 1, 2017.

[22] S. E. Bibri, Big Data Science and Analytics for Smart Sustainable Urbanism: Unprecedented Paradigmatic Shifts and Practical Advancements. Springer, Cham, 2019.
[23] S. S. Dawes, A. M. Cresswell, and T. A. Pardo, "From 'Need to Know' to 'Need to Share': Tangled Problems, Information Boundaries, and the Building of Public Sector Knowledge Networks,” Public Adm. Rev., vol. 69, no. 3, pp. 392-402, May 2009, Accessed: Oct. 19, 2015. [Online].

[24] M. Janssen and N. Helbig, "Innovating and changing the policy-cycle: Policy-makers be prepared!," Gov. Inf. Q., vol. 35, no. 4, Supplement, pp. S99-S105, Oct. 2018.

[25] K. Löfgren and W. Webster, "Big data in government: The case of 'smart cities,'” in Big Data, Edward Elgar Publishing, 2019.

[26] F. Haneem, N. Kama, N. Taskin, D. Pauleen, and N. A. Abu Bakar, "Determinants of master data management adoption by local government organizations: An empirical study," Int. J. Inf. Manage., vol. 45, pp. 25-43, Apr. 2019.

[27] S. A. Shah, D. Z. Seker, M. M. Rathore, S. Hameed, S. Ben Yahia, and D. Draheim, “Towards Disaster Resilient Smart Cities: Can Internet of Things and Big Data Analytics Be the Game Changers?,” IEEE Access, vol. 7, pp. 91885-91903, 2019.

[28] S. Raghavan, B. Y. L. Simon, Y. L. Lee, W. L. Tan, and K. K. Kee, "Data Integration for Smart Cities: Opportunities and Challenges,” in Computational Science and Technology, 2020, pp. 393-403.

[29] A. Al-Lawati and L. Barbosa, “A Framework for Intelligent Policy Decision Making Based on a Government Data Hub,” in Digital Transformation and Global Society, 2019, pp. 92-106.

[30] G. Shmueli and O. Koppius, "Predictive analytics in information systems research," Robert H. Smith School Research Paper No. RHS, pp. 06-138, 2010, Accessed: Dec. 04, 2016. [Online].

[31] S. LaValle, E. Lesser, R. Shockley, M. S. Hopkins, and N. Kruschwitz, "Big data, analytics and the path from insights to value," MIT sloan management review, vol. 52, no. 2, p. 21, 2011, Accessed: Feb. 19, 2017. [Online].

[32] P. Brous, M. Janssen, D. Schraven, J. Spiegeler, and B. Can Duzgun, "Factors Influencing Adoption of IoT for Data-driven Decision Making in Asset Management Organizations," in Proceedings of the 2nd International Conference on Internet of Things, Big Data and Security, Porto, Portugal, 2017, pp. 7079, Accessed: Apr. 14, 2020. [Online].

[33] P. Brous, P. M. Herder, and M. Janssen, "Governing Asset Management Data Infrastructures,” vol. 95, pp. 303-310, Dec. 2016, Accessed: Apr. 14, 2020. [Online].

[34] F. Henning, “A theoretical framework on the determinants of organisational adoption of interoperability standards in Government Information Networks,” Gov. Inf. Q., vol. 35, no. 4, Supplement, pp. S61-S67, Oct. 2018.

[35] O. Benfeldt, J. S. Persson, and S. Madsen, "Data Governance as a Collective Action Problem,” Inf. Syst Front., Apr. 2019, doi: 10.1007/s10796-019-09923-z.

[36] P. Brous, M. Janssen, and R. Krans, “Data Governance as Success Factor for Data Science,” in Responsible Design, Implementation and Use of 
Information and Communication Technology, 2020, pp. 431-442.

[37] R. Abraham, J. vom Brocke, and J. Schneider, "Data Governance: A conceptual framework, structured review, and research agenda,” vol. 49, Jul. 2019, doi: 10.1016/j.ijinfomgt.2019.07.008.

[38] P. Brous, M. Janssen, and R. Vilminko-Heikkinen, "Coordinating Decision-Making in Data Management Activities: A Systematic Review of Data Governance Principles,” in ResearchGate, Sep. 2016, doi: 10.1007/978-3-319-44421-5_9.

[39] S. Cuno, L. Bruns, N. Tcholtchev, P. Lämmel, and I. K. Schieferdecker, "Data Governance and Sovereignty in Urban Data Spaces Based on Standardized ICT Reference Architectures,” vol. 4, no. 1), p. 16, Jan. 2019, Accessed: Apr. 14, 2020. [Online].

[40] A. Gupta, P. Panagiotopoulos, and F. Bowen, "An orchestration approach to smart city data ecosystems," Technol. Forecast. Soc. Change, vol. 153, p. 119929, Apr. 2020.

[41] C. M. Daily, D. R. Dalton, and A. A. Cannella, "Corporate Governance: Decades of Dialogue and Data,” AMRO, vol. 28, no. 3, pp. 371-382, Jul. 2003.

[42] S. Funtowicz and J. Ravetz, "Post-Normal Science. Science and Governance under Conditions of Complexity,” in Interdisciplinarity in Technology Assessment: Implementation and its Chances and Limits, M. Decker and F. Wütscher, Eds. Berlin, Heidelberg: Springer Berlin Heidelberg, 2001, pp. 1524.

[43] D. S. Sayogo, J. R. Gil-Garcia, and F. Cronemberger, "Clarity of roles and responsibilities in interagency information sharing (IIS) projects: determinants and impact on success," International Journal of Electronic Governance, vol. 10, no. 3, pp. 296-316, 2018, Accessed: Apr. 11, 2020. [Online]

[44] V. Khatri and C. V. Brown, "Designing Data Governance,” Commun. ACM, vol. 53, no. 1, pp. 148152, Jan. 2010.

[45] N. Miloslavskaya and A. Tolstoy, "Big data, fast data and data lake concepts,” Procedia Comput. Sci., vol. 88, no. 300-305, p. 63, 2016.

[46] C. Madera and A. Laurent, "The next information architecture evolution: the data lake wave," in Proceedings of the 8th International Conference on Management of Digital EcoSystems, Biarritz, France, Nov. 2016, pp. 174-180, Accessed: Apr. 14, 2020. [Online].

[47] P. Kulkarni and K. B. Akhilesh, "Big Data Analytics as an Enabler in Smart Governance for the Future Smart Cities," in Smart Technologies : Scope and Applications, K. B. Akhilesh and D. P. F. Möller, Eds. Singapore: Springer Singapore, 2020, pp. 53-65.

[48] T. van den Broek and A. F. van Veenstra, "Governance of big data collaborations: How to balance regulatory compliance and disruptive innovation,” Technol. Forecast. Soc. Change, vol. 129, pp. 330-338, Apr. 2018.

[49] W. van der Aalst, "Data Science in Action,” in Process Mining: Data Science in Action, W. van der
Aalst, Ed. Berlin, Heidelberg: Springer Berlin Heidelberg, 2016, pp. 3-23.

[50] T. Priebe and S. Markus, "Business information modeling: A methodology for data-intensive projects, data science and big data governance,” in 2015 IEEE International Conference on Big Data (Big Data), Oct. 2015, pp. 2056-2065.

[51] C. Dremel, E. Stoeckli, and J. Wulf, "Management of analytics-as-a-service - results from an action design research project,” Mar. 2020, doi: 10.1080/2573234X.2020.1740616.

[52] S. Passi and S. J. Jackson, “Trust in Data Science: Collaboration, Translation, and Accountability in Corporate Data Science Projects,” presented at the Proceedings of the ACM on Human-Computer Interaction, Nov. 2018.

[53] M. H. Amini, A. Imteaj, and P. M. Pardalos, "Interdependent Networks: A Data Science Perspective,” Patterns Prejudice, vol. 1, no. 1, p. 100003, Apr. 2020.

[54] S. Cuganesan, A. Hart, and C. Steele, "Managing information sharing and stewardship for public-sector collaboration: a management control approach,” Public Management Review, vol. 19, no. 6, pp. 862879, Jul. 2017.

[55] J. R. Gil-Garcia and T. A. Pardo, "E-government success factors: Mapping practical tools to theoretical foundations,” Gov. Inf. Q., vol. 22, no. 2, pp. 187-216, 2005, Accessed: Nov. 23, 2016. [Online].

[56] A. J. Meijer, J. R. Gil-Garcia, and M. P. R. Bolívar, "Smart City Research: Contextual Conditions, Governance Models, and Public Value Assessment," Soc. Sci. Comput. Rev., vol. 34, no. 6, pp. 647-656, 2016, Accessed: Apr. 30, 2017. [Online].

[57] W. W. Cities, "Kansas City, Louisville, and Washington, DC, Level Up,” Medium, Apr. 24, 2019. https://medium.com/what-works-citiescertification/kansas-city-louisville-and-washington-dclevel-up-cef847c62a6f (accessed May 03, 2020).

[58] W. W. Cities, “Kansas City: Winning Residents' Trust By Asking, Listening, and Acting,” Medium, Jan. 25, 2018. https://medium.com/what-works-citiescertification/kansas-city-winning-residents-trust-byasking-listening-and-acting-e56f59dbb257 (accessed May 03, 2020).

[59] "How Kansas City cemented data-driven management into city law,” Medium, Mar. 06, 2019. https://medium.com/@BloombergCities/how-kansascity-cemented-data-driven-management-into-city-law413335d68e12 (accessed May 03, 2020).

[60] I. Tucker, J. R. Gil-Garcia, and D. S. Sayogo, "Collaborative Data Analytics for Emergency Response: Identifying Key Factors and Proposing a Preliminary Framework," in Proceedings of the 10th International Conference on Theory and Practice of Electronic Governance, New Delhi AA, India, 2017, pp. 508-515. 